\title{
Relationship between cognitive insight and subjective quality of life in outpatients with schizophrenia
}

\author{
Jong-Hoon Kim' \\ Seul Lee' \\ Ah-Young Han' \\ Kyungwook Kim² \\ Jinyoung Lee'
}

'Neuroscience Research Institute, Department of Psychiatry, Gil Medical Center, Gachon University School of Medicine, Gachon University, Incheon, Republic of Korea; ${ }^{2}$ Department of Medicine, Gachon University School of Medicine, Gachon University, Incheon, Republic of Korea
Correspondence: Jong-Hoon Kim Neuroscience Research Institute, Department of Psychiatry, Gil Medical Center, Gachon University School of Medicine, Gachon University, II 98 Guwol-dong, Namdong-gu, Incheon 405-760, Republic of Korea

Tel +82324602696

$\mathrm{Fax}+82324728813$

Email jhnp@chol.com
This article was published in the following Dove Press journal:

Neuropsychiatric Disease and Treatment

7 August 2015

Number of times this article has been viewed

Background: The concept of cognitive insight refers to the cognitive processes involved in patients' re-evaluation of their anomalous experiences and of their misinterpretations. The purpose of the present study was to examine the relationship between cognitive insight and subjective quality of life in patients with schizophrenia to further shed light on the nature of cognitive insight and its functional correlates in schizophrenia.

Methods: Seventy-one stable outpatients with schizophrenia were evaluated for cognitive insight and subjective quality of life using the Beck Cognitive Insight Scale (BCIS) and the Schizophrenia Quality of Life Scale Revision 4 (SQLS-R4). The symptoms of schizophrenia were also assessed. Pearson's correlation analysis and partial correlation analysis that controlled for the severity of symptoms were performed to adjust for the possible effects of symptoms. Results: The self-reflectiveness subscale score of the BCIS had significant positive correlations with the SQLS-R4 psychosocial domain and total SQLS-R4 scores, indicating that the higher the level of cognitive insight, the lower the subjective quality of life. In partial correlation analysis controlling for symptoms, the BCIS self-reflectiveness subscale score still had a significant correlation with the SQLS-R4 psychosocial domain score. The correlation coefficient between the BCIS self-reflectiveness and total SQLS-R4 scores was reduced to a nonsignificant statistical tendency.

Conclusion: The results of our study suggest that cognitive insight, particularly the level of self-reflectiveness, is negatively associated with the level of subjective quality of life in outpatients with schizophrenia and that this relationship is not wholly due to the confounding effect of symptoms. Future studies are necessary to explore possible mediating and moderating factors and to evaluate the effects of therapeutic interventions on the relationship.

Keywords: cognitive insight, self-reflectiveness, subjective quality of life, depressive symptoms, schizophrenia

\section{Introduction}

It has been reported that the lack of insight is prevalent among patients with schizophrenia and that impaired insight is significantly associated with medication noncompliance, frequent relapse, and poor prognosis. ${ }^{1-5}$ Insight in mental illness is generally a multidimensional concept that includes the following aspects: 1) awareness of the mental disorder, 2) understanding of the social consequences of the disorder, 3 ) awareness of the need for treatment, 4) awareness of specific signs and symptoms, and 5) the attribution of symptoms to the disorder. ${ }^{1-3,6}$

For patients with schizophrenia and related psychotic disorders, however, there has been a crucial extension to the concept of insight: it has been suggested that the cognitive processes involved in patients' re-evaluation of their anomalous experiences 
and of their misinterpretations should be incorporated into this concept. ${ }^{7}$ The Beck Cognitive Insight Scale (BCIS) has been developed and utilized to evaluate patients' selfreflectiveness and overconfidence in the interpretations of their experiences. ${ }^{7}$ The BCIS assesses patients' reports of their objectivity regarding their current anomalous thoughts, their perspective about previous errors, their capacity for reattribution of erroneous explanations, and their receptiveness to corrective information from other people. ${ }^{7,8}$ Previous studies have shown that cognitive insight is significantly disrupted in patients with schizophrenia compared with those with nonpsychotic disorders, such as mood disorders. ${ }^{7,9}$ This reflects the metacognitive deficits inherent in schizophrenia. ${ }^{10}$ A recent factor analytic study has found that the BCIS score loads with metacognitive awareness in patients with schizophrenia or schizoaffective disorder, indicating that cognitive insight is associated with the ability to form complex images of oneself and others, a synthetic form of metacognition. ${ }^{11}$

There have been reports on the clinical correlates of cognitive insight in schizophrenia. The disruption of cognitive insight has been linked to delusions, ${ }^{7,12,13}$ hallucinations, ${ }^{12}$ negative symptoms, ${ }^{14-16}$ and depressive/ anxiety symptoms, ${ }^{17,18}$ although not all studies observed such associations, ${ }^{16,19,20}$ and some inconsistencies have arisen. Only a few studies, in contrast, have examined the association between cognitive insight and functional outcome measures. Metacognitive deficit was found to be associated with impaired coping, ${ }^{10}$ and a low level of cognitive insight was related to poor social functioning ${ }^{19}$ and to lower global assessment of functioning scores. ${ }^{21}$

At present, however, the relationship between cognitive insight and subjective quality of life has not been explored. Moreover, the results of previous studies regarding the relationship between clinical, but not cognitive, insight and the subjective quality of life or well-being have been inconclusive. ${ }^{22,23}$ There have been suggestions that patients with better insight experience more severe depressive symptoms and may realize their restrictions more clearly, which decreases their subjective quality of life and overall sense of satisfaction. ${ }^{22-26}$

Considering previous reports that patients with high cognitive insight were more depressed and had received psychoeducation more often ${ }^{27}$ and that the increased awareness of self-perceived limitations may also lead to hopelessness, ${ }^{28}$ it can therefore be hypothesized that a higher level of cognitive insight, or self-reflectiveness, may be associated with more severe emotional distress and a lower subjective quality of life. Hence, in the present study, we investigated the relationship between cognitive insight and subjective quality of life in stable outpatients with schizophrenia to further shed light on the nature of cognitive insight and its functional correlates in schizophrenia.

\section{Materials and methods Subjects}

The study protocol was approved by the Institutional Review Board of the Gachon University Gil Medical Center, and all procedures used in the study were conducted in accordance with international ethical standards, Declaration of Helsinki.

The criteria for patient recruitment were the following: (i) a diagnosis of schizophrenia by the Diagnostic and Statistical Manual of Mental Disorders, 4th edition, ${ }^{29}$ which was established using the Structured Clinical Interview for Diagnostic and Statistical Manual of Mental Disorders, 4th edition; ${ }^{30}$ (ii) age between 20 and 50 years; (iii) outpatients on at least 4 weeks of maintenance therapy with atypical antipsychotics without changes in the dosage of medications over the same period; and (iv) be able to be rated reliably on psychiatric rating scales. Patients were excluded if they (i) met diagnostic criteria for a psychiatric diagnosis other than schizophrenia, (ii) had a concurrent diagnosis of substance abuse or dependence, (iii) had a history of head trauma with loss of consciousness, or (iv) had concurrent medical or neurological disorders.

Seventy-one outpatients (33 men and 38 women) were enrolled in the study. Informed consent was obtained from all subjects after a full explanation of the study procedure. The demographic and clinical characteristics of the subjects are presented in Table 1. The subjects had a mean (standard deviation [SD]) age of 35.9 (10.4) years and a mean (SD) duration of illness of 7.1 (2.8) years. The duration of illness was defined as the period between the time that first psychotic symptoms were reported and the time of enrollment in the present study. The mean (SD) years of education were 12.0 (2.1) years. All subjects were chronic patients with one or two relapses and were receiving maintenance treatment with atypical antipsychotics. Twenty-eight patients (39.4\%) had a single relapse and 43 patients $(60.6 \%)$ had two relapses. None of the patients had been taking antidepressants or mood stabilizers. The antipsychotics that patients were taking at the time of enrollment were paliperidone $(n=30$, mean dose: $6.3[\mathrm{SD}=2.7] \mathrm{mg} /$ day $)$, olanzapine $(\mathrm{n}=17$, mean dose: 10.9 [SD=5.3] mg/day), aripiprazole $(n=13$, mean dose: 9.0 [SD=4.8] mg/day), clozapine $(\mathrm{n}=7$, mean dose: $242.9[\mathrm{SD}=100.7] \mathrm{mg} /$ day $)$, and quetiapine ( $\mathrm{n}=4$, mean dose: $337.5[\mathrm{SD}=85.3] \mathrm{mg} /$ day). The mean duration of current 
Table I Demographic and clinical characteristics of the subjects $(\mathrm{N}=7 \mathrm{I})$

\begin{tabular}{ll}
\hline Variables & $\begin{array}{l}\text { Mean } \pm \text { SD/number } \\
\text { (percentage) }\end{array}$ \\
\hline Age (years) & $35.9 \pm 10.4$ \\
Sex & \\
$\quad$ Male & $33(46.5 \%)$ \\
Female & $38(53.5 \%)$ \\
Duration of illness (years) & $7.1 \pm 2.8$ \\
Education (years) & $12.0 \pm 2.1$ \\
Marital status & \\
Single & $36(50.7 \%)$ \\
$\quad$ Married & $35(49.3 \%)$ \\
Manchester scale score & \\
Positive symptom & $2.6 \pm 2.8$ \\
Negative symptom & $3.4 \pm 3.2$ \\
CDSS score & $5.7 \pm 5.4$ \\
BCIS score & \\
Self-reflectiveness & $20.3 \pm 4.0$ \\
Self-certainty & $13.3 \pm 2.9$ \\
Composite index & $7.0 \pm 4.1$ \\
SQLS-R4 score & \\
Psychosocial domain & $45.1 \pm 18.5$ \\
Vitality domain & $30.4 \pm 9.6$ \\
Total score & $75.5 \pm 26.9$ \\
\hline
\end{tabular}

Abbreviations: $\mathrm{BCIS}$, Beck Cognitive Insight Scale; CDSS, Calgary Depression Scale for Schizophrenia; SD, standard deviation; SQLS-R4, Schizophrenia Quality of Life Scale Revision 4.

antipsychotic treatment was $2.3(\mathrm{SD}=2.1)$ years. Regarding concomitant medications, 21 patients $(29.6 \%)$ were administered both benzodiazepines and anticholinergics, nine patients (12.7\%) were administered anticholinergics alone, and 21 patients $(29.6 \%)$ were administered benzodiazepines only. The mean dosages of benzodiazepines and anticholinergics were $1.1(\mathrm{SD}=0.7) \mathrm{mg} /$ day (lorazepam equivalents) and 1.5 $(\mathrm{SD}=0.5) \mathrm{mg} /$ day (benztropine equivalents), respectively.

\section{Assessments}

Cognitive insight was assessed using the BCIS, ${ }^{7}$ which was developed to evaluate patients' self-reflectiveness and their overconfidence in their interpretations of their experiences. The BCIS is a 15-item self-report questionnaire with a 9-item self-reflectiveness subscale and a 6-item self-certainty subscale. Each item is rated on a 4-point scale from 0 (do not agree at all) to 3 (agree completely), and two subscale scores are obtained. A composite index is also obtained by subtracting the self-certainty subscale score from the selfreflectiveness subscale score. A Korean version of the BCIS was developed ${ }^{31,32}$ and used in this study.

Patients' subjective quality of life was assessed using the Schizophrenia Quality of Life Scale Revision 4 (SQLS-R4). ${ }^{33}$
The SQLS-R4 is a self-administered scale comprising 33 items in two domains, that is, psychosocial and vitality. The SQLS-R4 is a schizophrenia-specific quality of life instrument and it measures quality of life from the patients' own perspective. All except four items are scored on a 5 -point Likert-type scale $(0=$ never, $1=$ rarely, $2=$ sometimes, $3=$ often, and $4=$ always), with the exceptional four items being reverse coded $(0=$ always, $1=$ often, $2=$ sometimes, $3=$ rarely, and $4=$ never). The scores for the two domains and total scores are obtained. A lower score represents a better quality of life, while a higher score indicates a lower quality of life. ${ }^{33-36}$ Previous studies have shown sufficient internal consistency and construct validity of the SQLS-R4. ${ }^{34-36}$ The psychometric properties of the Korean version of the SQLS-R4 were previously examined, ${ }^{36}$ which was used in this study. The SQLS-R4 has been increasingly used in different populations of patients with schizophrenia. ${ }^{37,38}$

The symptoms of schizophrenia were evaluated using the Manchester Scale (MS). ${ }^{39}$ The MS is a widely used clinician-rated instrument for assessing psychiatric symptoms in schizophrenia and scores are summed across items rated on a 5-point Likert scale. ${ }^{39-42}$ It consists of eight items measuring a broad range of symptoms: delusion, hallucination, incoherence, flattened affect, poverty of speech, psychomotor retardation, depression, and anxiety. The positive symptom score is defined as the sum score of delusion, hallucination, and incoherence items, and the negative symptom score is indicated by the sum score of flattened affect, poverty of speech, and psychomotor retardation. The depression/anxiety symptom score represents the sum score of depression and anxiety items and is one of the important dimensions of general psychopathology in schizophrenia. ${ }^{39-46}$ The severity of depressive symptoms was also assessed using the Calgary Depression Scale for Schizophrenia (CDSS). ${ }^{47}$ The CDSS is the most widely used clinician-rated scale for assessing depression in schizophrenia. ${ }^{47,48}$

Each patient had a baseline interview and was evaluated using the MS and the CDSS by an investigator who was sufficiently trained and familiar with the assessment of psychiatric symptoms in schizophrenia ${ }^{46,49,50}$ and was assessed using the BCIS and the SQLS-R4 in the presence of a research assistant who monitored whether or not the questions were completely understood.

\section{Statistical analysis}

The primary measures for analysis were the BCIS scores and the SQLS-R4 scores. Pearson's correlation analyses between BCIS scores and SQLS-R4 scores were performed. 
The analyses were conducted for both total and subscale scores. Pearson's partial correlation analyses were also performed between BCIS scores and SQLS-R4 scores, controlling for the severity of symptoms, to adjust for the possible effects of symptoms and to exclusively assess the correlation between cognitive insight and subjective quality of life. For all analyses, the level of statistical significance was defined as $P<0.05$ (two-tailed). All statistical analyses were performed using SPSS 19.0 for windows (SPSS, Chicago, IL).

\section{Results}

The intercorrelations between the BCIS scores and clinical variables are presented in Table 2. The BCIS scores had no significant correlations with age ( $r=-0.17$ to $0.09, P>0.05$ ), $\operatorname{sex}(\rho=-0.21$ to $0.06, P>0.05)$, duration of illness $(r=-0.14$ to $0.04, P>0.05)$, number of relapses $(r=-0.18$ to -0.10 , $P>0.05)$, years of education ( $r=-0.22$ to $0.11, P>0.05$ ), or marital status ( $\rho=-0.11$ to $0.05, P>0.05$ ). The type of antipsychotics, which patients were taking at the time of the assessment, was not significantly associated with the BCIS scores ( $\rho=-0.21$ to $-0.05, P>0.05$ ). The BCIS scores had no significant correlations with the duration of current antipsychotic treatment $(r=-0.04$ to $-0.01, P>0.05$ ) or the use of concomitant medications ( $\rho=-0.01$ to $-0.03, P>0.05$ ). With respect to the MS scores, the BCIS scores had no significant

Table 2 Correlation coefficients between cognitive insight and demographic/clinical variables

\begin{tabular}{llll}
\hline Variables & $\begin{array}{l}\text { BCIS SR } \\
\text { score }\end{array}$ & $\begin{array}{l}\text { BCIS SC } \\
\text { score }\end{array}$ & $\begin{array}{l}\text { BCIS CI } \\
\text { score }\end{array}$ \\
\hline Age & -0.11 & 0.09 & -0.17 \\
Sex & -0.13 & 0.06 & -0.21 \\
Duration of illness & -0.11 & 0.04 & -0.14 \\
Number of relapse & -0.18 & -0.10 & -0.11 \\
Education & -0.05 & -0.22 & 0.11 \\
Marital status & -0.03 & 0.05 & -0.11 \\
Antipsychotics & -0.21 & -0.14 & -0.05 \\
Duration of current & -0.04 & -0.01 & -0.03 \\
antipsychotic treatment & & & \\
Use of concomitant medications & 0.03 & -0.01 & 0.03 \\
Manchester scale & & & \\
$\quad$ Positive symptom score & 0.07 & -0.15 & 0.18 \\
$\quad$ Negative symptom score & 0.07 & 0.02 & 0.06 \\
Depression/anxiety symptom & -0.07 & -0.07 & -0.02 \\
score & & & \\
CDSS score & 0.12 & -0.05 & 0.16 \\
\hline N & &
\end{tabular}

Notes: Data are presented as Spearman's rank correlation coefficients ( $\rho$, twotailed; for sex, marital status, antipsychotics, and the use of concomitant medications) and Pearson's correlation coefficients ( $r$, two-tailed; for the other variables). ${ }^{\mathrm{a}}$ The type of antipsychotics that patients were taking at the time of the assessment.

Abbreviations: BCIS, Beck Cognitive Insight Scale; CDSS, Calgary Depression Scale for Schizophrenia; Cl, composite index; SC, self-certainty; SR, self-reflectiveness. correlations with the positive symptom score $(r=-0.15$ to $0.18, P>0.05$ ), negative symptom score ( $r=0.02$ to 0.07 , $P>0.05)$, or depression/anxiety symptom score ( $r=-0.07$ to $-0.02, P>0.05$; Table 2). In addition, there were no significant correlations between the BCIS scores and the CDSS score ( $r=-0.05$ to $0.16, P>0.05$; Table 2 ).

Table 3 shows the intercorrelations between the SQLS-R4 scores and clinical variables.

The SQLS-R4 scores had no significant correlations with age ( $r=-0.09$ to $0.03, P>0.05$ ), $\operatorname{sex}(\rho=-0.11$ to -0.08 , $P>0.05$ ), duration of illness ( $r=-0.10$ to $-0.02, P>0.05$ ), number of relapses ( $r=-0.09$ to $0.01, P>0.05$ ), years of education ( $r=-0.08$ to $-0.01, P>0.05$ ), or marital status ( $\rho=-0.04$ to $-0.01, P>0.05$ ). The type of antipsychotics was not significantly associated with the SQLS-R4 scores ( $\rho=-0.14$ to -0.07 , $P>0.05$ ). The SQLS-R4 scores had no significant correlations with the duration of current antipsychotic treatment $(r=-0.05$ to $-0.02, P>0.05$ ) or the use of concomitant medications $(\rho=-0.09$ to $-0.07, P>0.05$ ).

With respect to the MS scores, the SQLS-R4 scores had no significant correlations with the positive symptom score ( $r=0.19$ to $0.20, P>0.05)$ or negative symptom score $(r=0.19$ to $0.22, P>0.05)$. However, the SQLS-R4 scores showed

Table 3 Correlation coefficients between subjective quality of life and demographic/clinical variables

\begin{tabular}{llll}
\hline Variables & $\begin{array}{l}\text { SQLS-R4 } \\
\text { psychosocial } \\
\text { domain score }\end{array}$ & $\begin{array}{l}\text { SQLS-R4 } \\
\text { vitality } \\
\text { domain score }\end{array}$ & $\begin{array}{l}\text { SQLS- } \\
\text { R4 total }\end{array}$ \\
\hline score
\end{tabular}

Notes: Data are presented as Spearman's rank correlation coefficients ( $\rho$, twotailed; for sex, marital status, antipsychotics, and the use of concomitant medications) and Pearson's correlation coefficients ( $r$, two-tailed; for the other variables). ${ }^{\text {TT The }}$ type of antipsychotics that patients were taking at the time of the assessment. $* * p<0.01$.

Abbreviations: CDSS, Calgary Depression Scale for Schizophrenia; SQLS-R4, Schizophrenia Quality of Life Scale Revision 4. 
Table 4 Correlations of cognitive insight with subjective quality of life

\begin{tabular}{llll}
\hline Variables & $\begin{array}{l}\text { BCIS SR } \\
\text { score }\end{array}$ & $\begin{array}{l}\text { BCIS SC } \\
\text { score }\end{array}$ & $\begin{array}{l}\text { BCIS CI } \\
\text { score }\end{array}$ \\
\hline SQLS-R4 psychosocial domain score & $0.31^{* *}$ & 0.10 & $0.23^{\ddagger}$ \\
SQLS-R4 vitality domain score & 0.09 & -0.01 & 0.10 \\
SQLS-R4 total score & $0.24^{*}$ & 0.07 & 0.20 \\
\hline
\end{tabular}

Notes: Data are presented as Pearson's correlation coefficients ( $r$, two-tailed). $* P<0.05, * * P<0.01,{ }^{\ddagger} P<0.1$.

Abbreviations: $\mathrm{BCIS}$, Beck Cognitive Insight Scale; $\mathrm{Cl}$, composite index; SC, self-certainty; SR, self-reflectiveness; SQLS-R4, Schizophrenia Quality of Life Scale Revision 4

significant correlations with depression/anxiety symptom score ( $r=0.36$ to $0.43, P<0.01$; Table 3 ). In addition, there were significant correlations between the SQLS-R4 scores and the CDSS score ( $r=0.50$ to $0.58, P<0.01$; Table 3 ).

With regard to the correlations between BCIS and SQLS-R4 scores, the self-reflectiveness subscale score of the BCIS had significant positive correlations with the SQLS-R4 psychosocial subscale $(r=0.31, P<0.01)$ and total SQLS-R4 scores $(r=0.24, P=0.04)$, indicating that the higher the level of cognitive insight, the lower the subjective quality of life (Table 4). There was also a tendency toward a positive correlation between the BCIS composite index and the SQLS-R4 psychosocial subscale score ( $r=0.23, P=0.05$; Table 4).

Since only the MS depression/anxiety and CDSS scores significantly correlated with the SQLS-R4 scores, we performed partial correlation analyses between BCIS scores and SQLS-R4 scores, controlling for the MS depression/anxiety and CDSS scores. The BCIS self-reflectiveness subscale score still had a significant positive correlation with the SQLS-R4 psychosocial domain score $(r=0.30, P=0.01$; Table 5), indicating that the strength of the association remained unchanged. However, the correlation coefficient between the BCIS self-reflectiveness subscale score and the total SQLS-R4 score was reduced to a nonsignificant statistical tendency $(r=0.22, P=0.08$; Table 5).

Table 5 Partial correlations between cognitive insight and subjective quality of life

\begin{tabular}{llll}
\hline Variables & BCIS & BCIS & BCIS \\
& SR score & SC score & CI score \\
\hline SQLS-R4 psychosocial domain score & $0.30^{*}$ & 0.16 & 0.18 \\
SQLS-R4 vitality domain score & 0.03 & 0.01 & 0.02 \\
SQLS-R4 total score & $0.22^{\ddagger}$ & 0.11 & 0.13 \\
\hline
\end{tabular}

Notes: Data are presented as partial correlation coefficients ( $r$, two-tailed), controlling for the Manchester Scale (MS), and Calgary Depression Scale for Schizophrenia (CDSS) scores. ${ }^{*} P<0.05,{ }^{\ddagger} P<0$. I.

Abbreviations: $\mathrm{BCIS}$, Beck Cognitive Insight Scale; $\mathrm{Cl}$, composite index; SC, self-certainty; SR, self-reflectiveness; SQLS-R4, Schizophrenia Quality of Life Scale Revision 4.

\section{Discussion}

In the present study, we examined the relationship between cognitive insight and subjective quality of life in stable outpatients with schizophrenia. The main finding was the significant relationship between the scores of BCIS selfreflectiveness subscale and SQLS-R4 psychosocial domain even after controlling for the MS and CDSS scores. To the best of our knowledge, this is among the first reports to explore the relationship between cognitive insight and subjective quality of life.

In Pearson's correlation analyses, the BCIS selfreflectiveness subscale score had significant positive correlations with the SQLS-R4 psychosocial subscale and total SQLS-R4 scores. The BCIS composite index also had a tendency to be positively correlated with the SQLS-R4 psychosocial subscale score. Since a lower SQLS-R4 score represents a better quality of life and a higher score indicates a lower quality of life, ${ }^{33-36,51}$ these results indicate that there is an inverse relationship between the level of cognitive insight and the subjective quality of life. Our findings are in line with previous suggestions that the awareness of anomalous experiences and misinterpretation and the inability to objectively evaluate them may lead to significant subjective discomfort. ${ }^{28,52}$ Our results are also in accordance with the findings of Giusti et $\mathrm{al}^{53}$ who showed that the self-report of being in recovery was significantly correlated with low self-reflectiveness of cognitive insight in patients with schizophrenia.

Notably, after controlling for the MS depression/anxiety and CDSS scores, the partial correlation between the BCIS self-reflectiveness subscale and SQLS-R4 psychosocial domain scores remained significant, while the correlation between self-reflectiveness and the total SQLS-R4 score did not. These results suggest that even though the severity of depression/anxiety symptoms may affect the relationship between cognitive insight and subjective quality of life to some degree, the correlation between the self-reflectiveness aspect of cognitive insight and the psychosocial domain of subjective quality of life has unique variance that is not due to depression/anxiety symptoms. There were previous reports that awareness of illness was associated with poor subjective quality of life, even when co-varying for the Brief Psychiatric Rating Scale ${ }^{54}$ depression item. ${ }^{24}$ Our results may extend these findings to include cognitive insight that is conceptually relevant to metacognition. ${ }^{10}$ One possible explanation for these findings is that internalized stigma-related variables may moderate the relationship between cognitive insight and subjective quality of life. ${ }^{55}$ A second possibility is that personality characteristics or symptoms other than 
depression/anxiety may mediate or moderate the relationship between the two.$^{23}$ Furthermore, as previously suggested by Lysaker et $\mathrm{al}^{11}$ self-reflectivity is closely related to the ability to synthesize or integrate discrete judgments into integrated wholes and to form complex ideas about oneself and monitor them over time. As such, greater awareness could leave vulnerable persons open to perceiving things that are deeply unsettling, which may result in current and prospective emotional discomfort and poor interpersonal relationships. ${ }^{56}$

It should be noted that the SQLS-R4 scores had significant correlations with the MS depression/anxiety score and the CDSS score. These results are clearly in line with previous reports that psychiatric symptoms, particularly general psychopathology, are significantly inversely related to quality of life. ${ }^{57,58}$ In addition, our findings are consistent with previous findings that depressive mood and anxiety are significantly associated with a low quality of life in schizophrenia. ${ }^{59,60}$

In our study, the MS positive or negative symptoms were not significantly correlated with the BCIS or SQLS-R4 scores; therefore, they were not controlled in the partial correlation analysis. In contrast, the MS depression/anxiety and CDSS scores were significantly correlated with the SQLS-R4 scores and were controlled in the analysis. However, as previously reported by Lincoln et $\mathrm{al}^{61}$ psychiatric symptom severity is an important mediator in the long-term relationship between insight and functional outcome. Therefore, prospective studies on the relationship between cognitive insight and subjective quality of life are required using a more thorough assessment of psychopathology with different comprehensive scales.

In the present study, the depression/anxiety symptom score was significantly correlated with both domains of the SQLS-R4, while it was not associated with the self-reflectiveness score. Considering that the self-reflectiveness score had a significant correlation with the SQLS-R4 psychosocial domain score, our findings suggest that self-reflectivity and depression/anxiety symptoms may have additive effects on the psychosocial domain of subjective quality of life.

The results of our study also has an implication that careful individualized approaches focused on coping strategies may be required to enhance both cognitive insight and subjective quality of life. The therapeutic approaches that assist patients to develop more complex accounts of their lives, to develop integrated and realistic ideas about their own identities, and to use these representations to understand and effectively respond to psychological and interpersonal challenges are to be emphasized. ${ }^{62}$
It should be noted that we used a self-report quality of life scale. Previous studies have shown that self-report and observer-rated quality of life instruments often have different determinants ${ }^{63}$ and that the correlations between the two range from low to moderate. ${ }^{64,65}$ Thus, if an observer-rated quality of life scale had been used in our study, different results might have been found. In a previous longitudinal study, ${ }^{66}$ better insight, as assessed by the item G12 from the Positive and Negative Symptom Scale for Schizophrenia, ${ }^{67}$ was significantly correlated with improved social functioning measured by an observer-rated scale. Patients with higher cognitive insight were also more likely to live independently. ${ }^{19}$ The self-report quality of life assessment is considered to have the advantage of gathering information on specific aspects conveniently and of understanding those aspects on the part of patients; ${ }^{68,69}$ however, it is not complemented by objective assessments of socio-occupational functioning. Therefore, future studies should include observer-rated scales, in addition to self-report instruments, to capture the multidimensional domains of quality of life in patients with schizophrenia.

The interpretation of the results of the present study should be considered in light of some limitations. This was a cross-sectional study in a naturalistic clinical setting. A prospective study with a larger sample is warranted to confirm the stability of the relationships that were observed..$^{70}$ The subjects were all outpatients receiving stable doses of antipsychotics; as such, the findings may not be generalized to a more diverse group of patients. The number of relapses and the medication status were not associated with the BCIS or SQLS-R4 scores; therefore, they were not considered to be confounding factors. Future studies should include first-episode or drug-naïve patients to evaluate whether the relationship observed in the present study holds true for those patients and to obtain valuable insights into the complex nature of the relationship between cognitive insight and quality of life. As previously mentioned, we used a self-rating scale to assess subjective quality of life. The use of an observer-rated quality of life scale might have led to different results.

\section{Conclusion}

The results of our study suggest that cognitive insight, particularly the level of self-reflectiveness, is significantly negatively associated with the subjective quality of life and that this relationship is not wholly due to the confounding effect of depressive symptoms. The findings in our study also suggest that careful individualized approaches focused 
on coping strategies may be required to enhance both cognitive insight and the subjective quality of life in patients with schizophrenia. Future studies are necessary to explore possible mediating and moderating factors, for example, neurocognitive function, personality traits, or self-stigma, and to evaluate the effects of pharmacological and nonpharmacological interventions on the relationship.

\section{Acknowledgments}

This study was supported by a grant of the Korean Health Technology R\&D Project, Ministry of Health \& Welfare, Republic of Korea (HI14C1731) and by the Gachon University Research Fund of 2015 (GCU-2015-5031).

\section{Disclosure}

The authors report no conflicts of interest in this work.

\section{References}

1. Amador XF, Strauss DH, Yale SA, Flaum MM, Endicott J, Gorman JM. Assessment of insight in psychosis. Am J Psychiatry. 1993;150(6): 873-879.

2. Amador XF, Flaum M, Andreasen NC, et al. Awareness of illness in schizophrenia and schizoaffective and mood disorders. Arch Gen Psychiatry. 1994;51(10):826-836.

3. BuckleyPF, WirshingDA, BhushanP, Pierre JM, ResnickSA, WirshingWC. Lack of insight in schizophrenia: impact on treatment adherence. CNS Drugs. 2007;21(2):129-141.

4. Pini S, Cassano GB, Dell'Osso L, Amador XF. Insight into illness in schizophrenia, schizoaffective disorder, and mood disorders with psychotic features. Am J Psychiatry. 2001;158(1):122-125.

5. Goldberg RW, Green-Paden LD, Lehman AF, Gold JM. Correlates of insight in serious mental illness. J Nerv Ment Dis. 2001;189(3): 137-145.

6. Mintz AR, Dobson KS, Romney DM. Insight in schizophrenia: a metaanalysis. Schizophr Res. 2003;61(1):75-88.

7. Beck AT, Baruch E, Balter JM, Steer RA, Warman DM. A new instrument for measuring insight: the Beck Cognitive Insight Scale. Schizophr Res. 2004;68(2-3):319-329.

8. Riggs SE, Grant PM, Perivoliotis D, Beck AT. Assessment of cognitive insight: a qualitative review. Schizophr Bull. 2012;38(2):338-350.

9. Kao YC, Liu YP. The Beck Cognitive Insight Scale (BCIS): translation and validation of the Taiwanese version. BMC Psychiatry. 2010;10:27.

10. Lysaker PH, Olesek KL, Warman DM, et al. Metacognition in schizophrenia: correlates and stability of deficits in theory of mind and selfreflectivity. Psychiatry Res. 2011;190(1):18-22.

11. Lysaker PH, Gumley A, Luedtke B, et al. Social cognition and metacognition in schizophrenia: evidence of their independence and linkage with outcomes. Acta Psychiatr Scand. 2013;127(3):239-247.

12. Engh JA, Friis S, Birkenaes AB, et al. Delusions are associated with poor cognitive insight in schizophrenia. Schizophr Bull. 2010;36(4): 830-835.

13. Buchy L, Malla A, Joober R, Lepage M. Delusions are associated with low self-reflectiveness in first-episode psychosis. Schizophr Res. 2009; 112(1-3):187-191.

14. Bora E, Erkan A, Kayahan B, Veznedaroglu B. Cognitive insight and acute psychosis in schizophrenia. Psychiatry Clin Neurosci. 2007;61(6): 634-639.

15. Pedrelli P, McQuaid JR, Granholm E, et al. Measuring cognitive insight in middle-aged and older patients with psychotic disorders. Schizophr Res. 2004;71(2-3):297-305.
16. Tranulis $\mathrm{C}$, Lepage $\mathrm{M}$, Malla A. Insight in first episode psychosis: who is measuring what? Early Interv Psychiatry. 2008;2(1):34-41.

17. Colis MJ, Steer RA, Beck AT. Cognitive insight in inpatients with psychotic, bipolar, and major depressive disorders. J Psychopathol Behav Assess. 2006;28(4):242-249.

18. Warman DM, Lysaker PH, Martin JM. Cognitive insight and psychotic disorder: the impact of active delusions. Schizophr Res. 2007;90(1-3): 325-333.

19. Favrod J, Zimmermann G, Raffard S, Pomini V, Khazaal Y. The Beck Cognitive Insight Scale in outpatients with psychiatric disorders: further evidence from a French-speaking sample. Can J Psychiatry. 2008; 53(11):783-787.

20. Uchida T, Matsumoto K, Kikuchi A, et al. Psychometric properties of the Japanese version of the Beck Cognitive Insight Scale: relation of cognitive insight to clinical insight. Psychiatry Clin Neurosci. 2009; 63(3):291-297.

21. O'Connor JA, Wiffen B, Diforti M, et al. Neuropsychological, clinical and cognitive insight predictors of outcome in a first episode psychosis study. Schizophr Res. 2013;149(1-3):70-76.

22. Karow A, Pajonk FG. Insight and quality of life in schizophrenia: recent findings and treatment implications. Curr Opin Psychiatry. 2006; 19(6):637-641.

23. Drake RJ. Insight into illness: impact on diagnosis and outcome of nonaffective psychosis. Curr Psychiatry Rep. 2008;10(3):210-216.

24. Hasson-Ohayon I, Kravetz S, Roe D, David AS, Weiser M. Insight into psychosis and quality of life. Compr Psychiatry. 2006;47(4): 265-269.

25. Karow A, Pajonk FG, Reimer J, et al. The dilemma of insight into illness in schizophrenia: self- and expert-rated insight and quality of life. Eur Arch Psychiatry Clin Neurosci. 2008;258(3):152-159.

26. Margariti M, Ploumpidis D, Economou M, Christodoulou GN, Papadimitriou GN. Quality of life in schizophrenia spectrum disorders: associations with insight and psychopathology. Psychiatry Res. 2015; 225(3):695-701

27. Misdrahi D, Denard S, Swendsen J, Jaussent I, Courtet P. Depression in schizophrenia: the influence of the different dimensions of insight. Psychiatry Res. 2014;216(1):12-16.

28. Schwartz RC. Self-awarensss in schizophrenia: its relationship to depressive symptomatology and broad psychiatric impairments. J Nerv Ment Dis. 2001;189(6):401-403.

29. American Psychiatric Association. Diagnostic and Statistical Manual of Mental Disorders. 4th ed. Washington DC: American Psychiatric Press; 1994.

30. First MB, Spitzer RL, Gibbon M, Williams JBW. Structured Clinical Interview for DSM-IV Axis I Disorders Research Version (SCID-I). New York: State Psychiatric Institute, Biometrics Research; 1996.

31. Kim YL, Youn T, Kim MS. A study on cognitive insight in patients with schizophrenia. Korean J Clin Psychol. 2006;25(4):1011-1027.

32. Kim HJ, Jhin HK, Chung EK, Chang D, Lee J. Crosscultural validation of the Beck Cognitive Insight Scale in Korean. Psychiatry Invest. 2007;4(2):109-115.

33. Wilkinson G, Hesdon B, Wild D, et al. Self-report quality of life measure for people with schizophrenia: the SQLS. Br J Psychiatry. 2000;177: $42-46$.

34. Kuo PJ, Chen-Sea MJ, Lu RB, et al. Validation of the Chinese version of the Schizophrenia Quality of Life Scale Revision 4 (SQLS-R4) in Taiwanese patients with schizophrenia. Qual Life Res. 2007;16(9): $1533-1538$

35. Martin CR, Allan R. Factor structure of the Schizophrenia Quality of Life Scale Revision 4 (SQLS-R4). Psychol Health Med. 2007;12(2): $126-134$.

36. Kim JH, Yim SJ, Min SK. The Korean Version of the 4th Revision of Schizophrenia Quality of Life Scale: validation study and relationship with PANSS. J Korean Neuropsychiatr Assoc. 2006;45(5):401-410.

37. Chou CY, Yang TT, Ma MC, Teng PR, Cheng TC. Psychometric validations and comparisons of schizophrenia-specific health-related quality of life measures. Psychiatry Res. 2015;226(1):257-263. 
38. Rouillon F, Eriksson L, Burba B, Raboch J, Kaprinis G, Schreiner A. Functional recovery results from the risperidone long-acting injectable versus quetiapine relapse prevention trial (ConstaTRE). Acta Neuropsychiatr. 2013;25(5):297-306.

39. Krawiecka M, Goldberg D, Vaughan M. A standardized psychiatric assessment scale for rating chronic psychotic patients. Acta Psychiatr Scand. 1977;55(4):299-308.

40. Manchanda R, Saupe R, Hirsch SR. Comparison between the Brief Psychiatric Rating Scale and the Manchester Scale for the rating of schizophrenic symptoms. Acta Psychiatr Scand. 1986;74(6):563-568.

41. Jackson HJ, Burgess PM, Minas IH, Joshua SD. Psychometric properties of the Manchester Scale. Acta Psychiatr Scand. 1990;81(2):108-113.

42. Barnes TRE, Nelson HE. The Assessment of Psychoses: A Practical Handbook. London: Chapman \& Hall Medical; 1994.

43. Lindenmayer JP, Grochowski S, Hyman RB. Five factor model of schizophrenia: replication across samples. Schizophr Res. 1995;14(3): 229-234.

44. Marder SR, Davis JM, Chouinard G. The effects of risperidone on the five dimensions of schizophrenia derived by factor analysis: combined results of the North American trials. J Clin Psychiatry. 1997;58(12): $538-546$.

45. Levine SZ, Rabinowitz J. Revisiting the 5 dimensions of the Positive and Negative Syndrome Scale. J Clin Psychopharmacol. 2007;27(5): 431-436.

46. Kim JH, Kim SY, Lee J, Oh KJ, Kim YB, Cho ZH. Evaluation of the factor structure of symptoms in patients with schizophrenia. Psychiatry Res. 2012;197(3):285-289.

47. Addington D, Addington J, Maticka-Tyndale E. Assessing depression in schizophrenia: the Calgary Depression Scale. Br J Psychiatry Suppl. 1993;22:39-44.

48. Addington J, Shah H, Liu L, Addington D. Reliability and validity of the Calgary Depression Scale for Schizophrenia (CDSS) in youth at clinical high risk for psychosis. Schizophr Res. 2014;153(1-3):64-67.

49. Kim JH, Cho SY, Byun HJ, Kang UG, Ahn YM, Kim YS. Multidimensional sensory phenomena in antipsychotic-induced akathisia. J Clin Psychopharmacol. 2004;24(6):618-623.

50. Kim JH, Son YD, Kim JH, et al. Serotonin transporter availability in thalamic subregions in schizophrenia: a study using 7.0-T MRI with [(11) C]DASB high-resolution PET. Psychiatry Res. 2015;231(1):50-57.

51. Taha NA, Ibrahim MI, Rahman AF, Shafie AA, Rahman AH. Validation of the schizophrenia quality of life scale revision 4 among chronic schizophrenia patients in Malaysia. Value in Health Reg Issues. 2012; 1(1):82-86.

52. Mohamed S, Rosenheck R, McEvoy J, Swartz M, Stroup S, Lieberman JA. Cross-sectional and longitudinal relationships between insight and attitudes toward medication and clinical outcomes in chronic schizophrenia. Schizophr Bull. 2009;35(2):336-346.

53. Giusti L, Ussorio D, Tosone A, et al. Is personal recovery in schizophrenia predicted by low cognitive insight? Community Ment Health J. 2015;51(1):30-37.

54. Overall JE, Gorham DR. The Brief Psychiatric Rating Scale. Psychol Rep. 1962;10:799-812.
55. Lysaker PH, Roe D, Yanos PT. Toward understanding the insight paradox: internalized stigma moderates the association between insight and social functioning, hope, and self-esteem among people with schizophrenia spectrum disorders. Schizophr Bull. 2007;33(1):192-199.

56. Buck B, Lysaker PH. Consummatory and anticipatory anhedonia in schizophrenia: stability, and associations with emotional distress and social function over six months. Psychiatry Res. 2013;205(1-2):30-35.

57. Eack SM, Newhill CE. Psychiatric symptoms and quality of life in schizophrenia: a meta-analysis. Schizophr Bull. 2007;33(5):1225-1237.

58. Eack SM, Newhill CE, Anderson CM, Rotondi AJ. Quality of life for persons living with schizophrenia: more than just symptoms. Psychiatr Rehabil J. 2007;30(3):219-222.

59. Huppert JD, Weiss KA, Lim R, Pratt S, Smith TE. Quality of life in schizophrenia: contributions of anxiety and depression. Schizophr Res. 2001;51(2-3):171-180.

60. Huppert JD, Smith TE. Longitudinal analysis of subjective quality of life in schizophrenia: anxiety as the best symptom predictor. J Nerv Ment Dis. 2001;189(10):669-675.

61. Lincoln TM, Lüllmann E, Rief W. Correlates and long-term consequences of poor insight in patients with schizophrenia. A systematic review. Schizophr Bull. 2007;33(6):1324-1342.

62. Lysaker PH, Buck KD, Carcione A, et al. Addressing metacognitive capacity for self reflection in the psychotherapy for schizophrenia: a conceptual model of the key tasks and processes. Psychol Psychother. 2011; 84(1):58-69.

63. Fitzgerald PB, Williams CL, Corteling N, et al. Subject and observerrated quality of life in schizophrenia. Acta Psychiatr Scand. 2001;103(5): 387-392.

64. Lobana A, Mattoo SK, Basu D, Gupta N. Quality of life in schizophrenia in India: comparison of three approaches. Acta Psychiatr Scand. 2001; 104(1):51-55.

65. Tomotake M, Kaneda Y, Iga J, et al. Subjective and objective measures of quality of life have different predictors for people with schizophrenia. Psychol Rep. 2006;99(2):477-487.

66. Saeedi H, Addington J, Addington D. The association of insight with psychotic symptoms, depression, and cognition in early psychosis: a 3-year follow-up. Schizophr Res. 2007;89(1-3):123-128.

67. Kay SR, Fiszbein A, Opler LA. The Positive and Negative Syndrome Scale (PANSS) for schizophrenia. Schizophr Bull. 1987;13(2): 261-276.

68. Naber D. A self-rating to measure subjective effects of neuroleptic drugs, relationships to objective psychopathology, quality of life, compliance and other clinical variables. Int Clin Psychopharmacol. 1995;10(Suppl 3):133-138.

69. de Haan L, Nimwegen L, Amelsvoort T, Dingemans P, Linszen D. Improvement of subjective well-being and enduring symptomatic remission, a 5-year follow-up of first episode schizophrenia. Pharmacopsychiatry. 2008;41(4):125-128.

70. Kako Y, Ito K, Hashimoto N, et al. The relationship between insight and subjective experience in schizophrenia. Neuropsychiatr Dis Treat. 2014;10:1415-1422.
Neuropsychiatric Disease and Treatment

\section{Publish your work in this journal}

Neuropsychiatric Disease and Treatment is an international, peerreviewed journal of clinical therapeutics and pharmacology focusing on concise rapid reporting of clinical or pre-clinical studies on a range of neuropsychiatric and neurological disorders. This journal is indexed on PubMed Central, the 'PsycINFO' database and CAS,

\section{Dovepress}

and is the official journal of The International Neuropsychiatric Association (INA). The manuscript management system is completely online and includes a very quick and fair peer-review system, which is all easy to use. Visit http://www.dovepress.com/testimonials.php to read real quotes from published authors. 